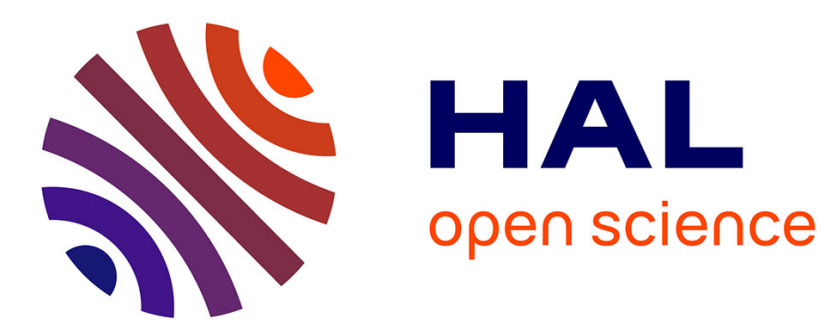

\title{
Doppler-free two-photon spectroscopy of neon. I. Fine structure and hyperfine constants for the $4 \mathrm{~d}$ ' subconfiguration
}

\author{
E. Giacobino, F. Biraben, G. Grynberg, B. Cagnac
}

\section{- To cite this version:}

E. Giacobino, F. Biraben, G. Grynberg, B. Cagnac. Doppler-free two-photon spectroscopy of neon.

I. Fine structure and hyperfine constants for the 4d' subconfiguration. Journal de Physique, 1977, 38

(6), pp.623-628. 10.1051/jphys:01977003806062300 . jpa-00208621

\section{HAL Id: jpa-00208621 \\ https://hal.science/jpa-00208621}

Submitted on 1 Jan 1977

HAL is a multi-disciplinary open access archive for the deposit and dissemination of scientific research documents, whether they are published or not. The documents may come from teaching and research institutions in France or abroad, or from public or private research centers.
L'archive ouverte pluridisciplinaire HAL, est destinée au dépôt et à la diffusion de documents scientifiques de niveau recherche, publiés ou non, émanant des établissements d'enseignement et de recherche français ou étrangers, des laboratoires publics ou privés. 


\title{
DOPPLER-FREE TWO-PHOTON SPECTROSCOPY OF NEON \\ I. FINE STRUCTURE AND HYPERFINE CONSTANTS FOR THE 4d' SUBCONFIGURATION
}

\author{
E. GIACOBINO, F. BIRABEN, G. GRYNBERG and B. CAGNAC \\ Laboratoire $\left({ }^{*}\right)$ de Spectroscopie Hertzienne de l'E.N.S. \\ Université Pierre-et-Marie-Curie, Tour 12, 75230 Paris Cedex 05, France
}

(Reçu le 21 décembre 1976, accepté le 14 février 1977)

\begin{abstract}
Résumé. - En utilisant la spectroscopie d'absorption à deux photons sans élargissement Doppler, nous avons mesuré la structure fine de la sous-configuration $4 \mathrm{~d}^{\prime}$ du néon ainsi que les constantes d'interaction hyperfine des quatre niveaux de cette sous-configuration dans le cas de l'isotope 21 . Nous décrivons le montage expérimental et nous insistons sur l'intérêt de placer la cellule d'expérience à l'intérieur d'une cavité Fabry-Pérot accordée à la longueur d'onde excitatrice. Nous comparons nos résultats expérimentaux sur les structures hyperfines avec les prédictions théoriques de Liberman et nous montrons qu'il y a un léger désaccord pour les niveaux de $J=2$. Nous expliquons ce désaccord en introduisant une constante d'interaction spin-orbite $\xi_{4 \mathrm{~d}}$ non nulle pour l'électron externe.
\end{abstract}

\begin{abstract}
Using Doppler-free two-photon spectroscopy, we have measured the fine structure of the $4 \mathrm{~d}^{\prime}$ subconfiguration of neon and the hyperfine constants of the four levels of this subconfiguration for the 21 isotope. We describe the experimental set-up and we point out the interest of putting the experimental cell inside a Fabry-Pérot cavity locked on the laser wavelength. We compare our experimental results for the hyperfine constants with the theoretical predictions of Liberman. We show that there is a slight disagreement for the two $J=2$ levels. This is explained by introducing a non zero spin orbit coupling $\xi_{4 \mathrm{~d}}$ for the external electron.
\end{abstract}

1. Introduction. - In two previous papers [1, 2], we have presented preliminary results concerning Doppler-free two-photon spectroscopy in Neon. Hyperfine structures in ${ }^{21} \mathrm{Ne}$ and isotope shifts $\left({ }^{20} \mathrm{Ne}-{ }^{21} \mathrm{Ne}-{ }^{22} \mathrm{Ne}\right)$ were determined in some of the levels of the $4 \mathrm{~d}$ configuration.

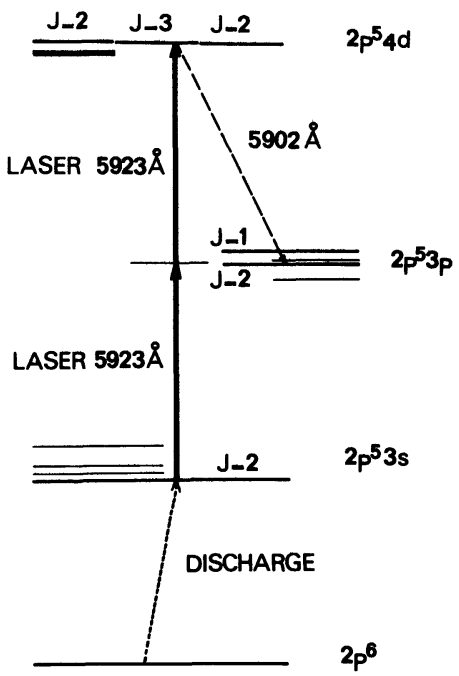

Fig. 1. - a) Simplified diagram of the energy levels of Neon.
Since that time other measurements have been performed in this configuration, in cases less favorable than those of the first experiments. Figure 1 gives a simplified energy diagram of Neon showing the concerned levels. These are the four levels of the $4 d^{\prime}$ subconfiguration labelled $4 d^{\prime}[3 / 2] 1,4 d^{\prime}[3 / 2] 2$,

(*) Associé au C.N.R.S.

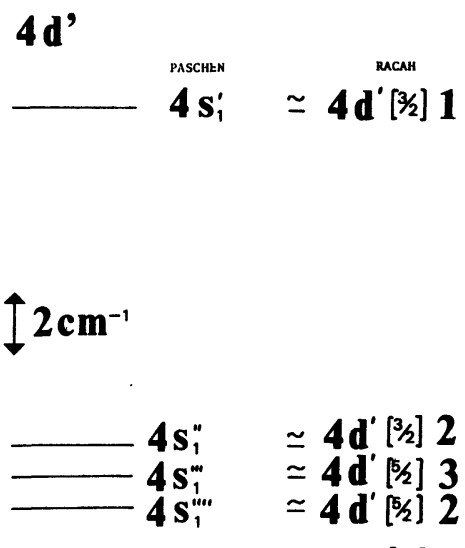

$[\mathbf{K}] \mathbf{J}$ 
$4 d^{\prime}$ [5/2] 3, 4d $45 / 2$ ] 2 in Racah notation (respectively $4 s_{1}^{\prime}, 4 s_{1}^{\prime \prime}, 4 s_{1}^{\prime \prime \prime}, 4 s_{1}^{\prime \prime \prime}$ in Paschen notation). An improved experimental set-up with the observed Neon cell inside a Fabry-Pérot cavity coupled with the laser cavity has enabled us to considerably increase the effective irradiating power. Thus we could obtain a fairly good signal-to-noise ratio on levels for which the two photon transition probability would be too small for observation with the less sophisticated setup.

As the isotope shift results for these levels have already been presented and discussed in ref. [2], we are going to deal here with fine and hyperfine structures; after describing the experimental set-up, we will give the results of our measurements and compare them to the values derived by a semi-empirical method [3]. We will show how comparison between theory and experiment lead us to propose an improvement to the theory.

2. Experimental set-up. - The main features of a Doppler-free two-photon experiment are now wellknown : the sample - here the neon cell - is irradiated by a monomode laser beam which is reflected back onto itself, so that an atom moving with axial velocity $v$ sees two waves with frequencies $\omega(1-v / c)$ and $\omega(1+v / c)$ ( $\omega$ being the laser frequency). Thus the condition for the atom to be excited from level $a$ to level $b$ by absorbing two photons (one from each wave) is, at resonance

$$
\omega_{\mathrm{b}}-\omega_{\mathrm{a}}=\omega(1-v / c)+\omega(1+v / c)=2 \omega
$$

which is independant of atomic velocity : the Doppler effect is eliminated.

Our experimental set-up (Fig. 2) involves a c.w. laser, the frequency of which is scanned by the means of servo-systems. The Spectra-Physics (model 580) dye laser pumped by a $4 \mathrm{WAr}^{+}$laser $(5145 \AA)$ can deliver about $150 \mathrm{~mW}$ for the resonance wavelengths (around $5920 \AA$ ) in monomode operation, which is enough for our experiments. Monomode running is provided by an internal Fabry-Pérot etalon, the thickness of which can be piezo-electrically varied.

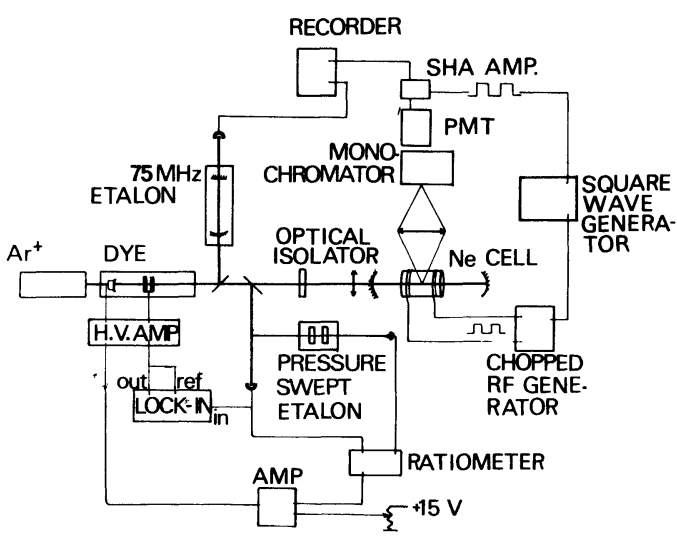

FIG. 2. - Experimental set-up.
One of the laser mirrors is also mounted on a piezoelectric transducer. Two servo-loops are used. The first one locks the frequency determined by the internal etalon on one mode of the laser cavity, using a modulation of the thickness of this etalon. The second one locks the length of the laser cavity on the side of the transmission peak of an external planar Fabry-Pérot etalon (free spectral range : $5 \mathrm{GHz}$ ) ; this loop involves comparison between the overall laser intensity and the intensity transmitted through the etalon, and does not necessitate any modulation. Due to the remaining jitter, the laser mode has a width of about $7 \mathrm{MHz}$ in a time of the order of a fraction of a second. The external etalon is thermally very stable, because of its cervit spacers; it is located inside a vacuum box ; letting air in through a calibrated leak provides pressure scanning of the laser frequency. Such a frequency scanning is almost linear; moreover the non-linear corrections can be easily calculated if necessary.

To calibrate the frequency scanning, a planar-focal etalon with a free spectral range of $75 \mathrm{MHz}$ is used. It is constructed with Invar and thermostated to $0.01^{\circ} \mathrm{C}$.

These stabilizing and monitoring devices only take a small part of the intensity. The main beam is focussed inside the experience cell using a $15 \mathrm{~cm}$ lens and reflected back on itself by a spherical mirror. An optical isolator is placed on the way of the laser beam to avoid coupling between the return beam and the laser cavity. We have used either a Faraday rotator, a glass rod (Sovirel, flint E0525) submitted to a convenient magnetic field when working with linear polarization, or a quarter-wave plate when circular polarization was needed. A further improvement consisted in making the laser beam pass several times through the cell : the cell is then placed inside a concentric resonator formed with two spherical mirrors; the rear mirror has a $99.5 \%$ reflectance the input mirror has a $94 \%$ reflectance. A $20 \mathrm{~cm}$ lens is used to match this cavity to the laser cavity. One of the mirrors is mounted on a piezoelectric ceramic. The length of the cavity is modulated and a servoloop is used to lock the cavity length so that the transmitted light is maximum. The interest of performing the experiment in such a cavity is clearly demonstrated in figure 3 , where we show recordings of the 3s $J=2 \rightarrow 4 \mathrm{~d}^{\prime} J=1$ two photon resonance without (Fig. $3 a$ ) and with the Fabry-Pérot resonator (Fig. 3b). The signal to noise ratio is increased by a factor of 15 .

The neon cell $\left({ }^{1}\right)$ has been sealed off after filling. As can be seen in figure $1 a$ the two-photon transitions concerned in the experiment do not start from the ground state but from a metastable state, that we must populate with a discharge. But the discharge brings atoms in all the other excited states too, making

$\left({ }^{1}\right)$ To minimize the reflection losses we use a cell with Brewsterangle windows; inside the Fabry-Pérot resonator we can only work with linear laser polarization. 


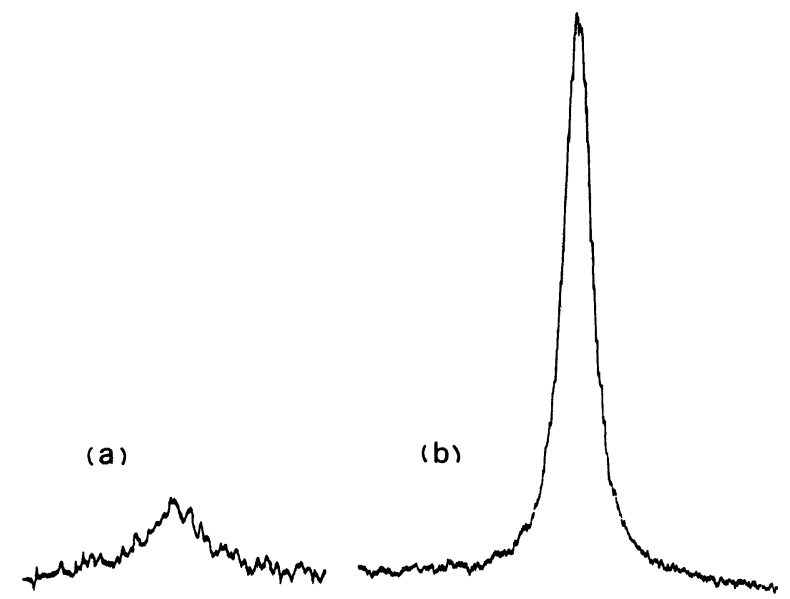

FIG. 3. - a) Recording of the 3s [3/2] 2 (metastable) $\rightarrow 4 d^{\prime}[3 / 2] 1$ two-photon transition without the Fabry-Pérot cavity. $b$ ) Recording of the same transition as in (a) with the cell inside the Fabry-Pérot cavity.

it impossible to detect the two-photon signal out of the fluorescence noise; so we had to work with an after-glow, where only the metastable states remain populated. A chopped discharge is supplied by a $14 \mathrm{MHz}$ generator, the output of which is modulated in square wave at a frequency of a few $\mathrm{kHz}$ (the lifetime of the metastable state is of the order of one millisecond in our cells). The fluorescence light emitted on one of the transitions from the excited state under investigation to a lower state is selected with a monochromator and detected with a photomultiplier. To eliminate the fluorescence signal of the discharge when it is on, the photomultiplier current is only transmitted during the after-glow, using a SHA 1 A (Analog Devices) amplifier. When sweeping the laser frequency the resulting signal is recorded as a function of time with a $3 \mathrm{~s}$ time-constant on the first trace of a double trace recorder. The transmission peaks given by a $75 \mathrm{MHz}$ reference etalon are simultaneously recorded on the second trace. Such a recording is shown in figure 4.
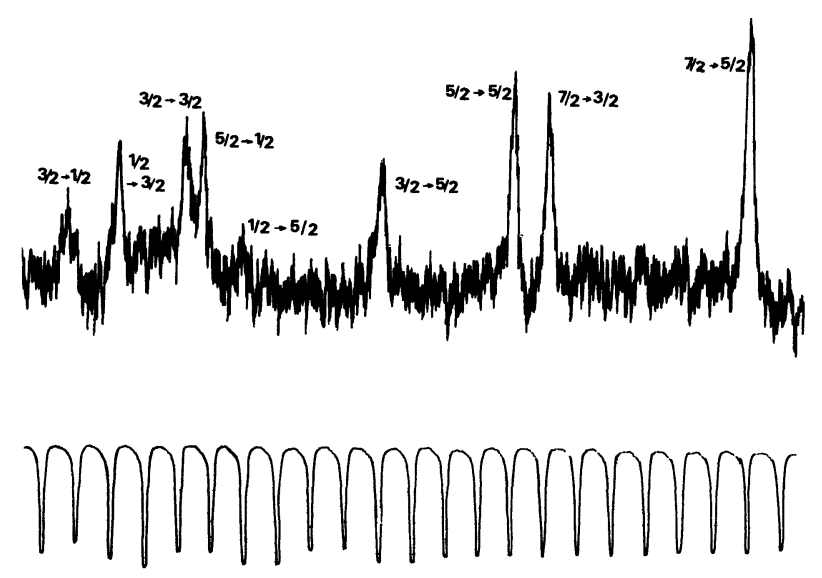

FIG. 4. - Recording of the hyperfine structure of the 3s [3/2] 2 (metastable) $\rightarrow 4 d^{\prime}[3 / 2] 1$ transition in ${ }^{21} \mathrm{Ne}$. The cell has been placed inside the Fabry-Pérot cavity.

3. Experimental results. - -3.1 FINE STRUCTURE INTERVALS. - The energy separations between the four levels of the $4 d^{\prime}$ subconfiguration are reported in table I. Let us point out that these results have been obtained on ${ }^{20} \mathrm{Ne}$ isotope with a natural neon pressure of 0.6 torr. Because of the specific mass-shift, the results would be different for ${ }^{22} \mathrm{Ne}$ [2]. On the other hand, one must take into account the pressure shift of the two-photon line due to interatomic collisions. The detailed investigation of these pressure shifts [5] has shown that they are nearly the same for the four $4 d^{\prime}$ levels; so we do not need to extrapolate the fine structure results to zero pressure.

TABLE I

Fine structure intervals in the $4 \mathrm{~d}^{\prime}$ subconfiguration for ${ }^{20} \mathrm{Ne}$

$4 d^{\prime}[5 / 2] 3-4 d^{\prime}[5 / 2] 2$ $4 d^{\prime}[3 / 2] 2-4 d^{\prime}[5 / 2] 3$ $4 d^{\prime}[3 / 2] 1-4 d^{\prime}[5 / 2] 3$

\begin{tabular}{cr}
\multicolumn{1}{c}{ This work } & Previous values [9] \\
- & - \\
$924.9 \pm 0.2 \mathrm{mK}$ & $926 \mathrm{mK}$ \\
$1051.6 \pm 0.2 \mathrm{mK}$ & $1049 \mathrm{mK}$ \\
$11869.2 \pm 1 \mathrm{mK}$ & $11857 \mathrm{mK}$
\end{tabular}

The last column of table I gives the values derived from $\mathrm{Ch}$. Moore's tables. It can be seen that the accuracy is considerably improved. Moreover, if the accuracy of the values from the spectroscopic tables is considered to be $1.0 \mathrm{mK}$, our values are not in agreement with them.

Because of the order of magnitude of these frequency intervals, it was not possible to cover them in a continuous scan of the laser frequency (the piezo-electric ceramic carrying the laser mirror only permits amplitudes of a few $\mathrm{GHz}$ ). The experiments has been done by sweeping the laser frequency over a small range around each two-photon resonance and by changing it by steps and counting the orders of convenient Fabry-Pérot etalons between.

3.2 Hyperfine STRUCTURe INTERVALS. - Using a cell containing $99 \%$ enriched ${ }^{21} \mathrm{Ne}$ isotope, we have recorded the various hyperfine components of the two-photon transitions. A typical recording is shown in figure 4. The hyperfine structure of the metastable $3 s$ [3/2] $2\left(1 s_{5}\right)$ level is well-known [6] and we have used it to verify the frequency calibration given by the Fabry-Pérot etalon. Nearly all the components are well separated. It was rather easy to identify them because, as shown in ref. [7], it is possible to predict their relative intensities, and because we already knew the order of magnitude of the hyperfine structures from ref. [3]. Table II shows the hyperfine intervals for the four investigated levels.

4. Interpretation of the hyperfine structures. 4.1 Calculation OF THE Dipolar MaGNETIC AND QUADRUPOLAR ELECTRIC CONSTANTS $A(\alpha J)$ AND $B(\alpha J)$. - As the hyperfine interaction is not very small 
TABLE II

\section{Hyperfine structure intervals} in the $4 \mathrm{~d}^{\prime}$ subconfiguration

$\begin{array}{cc}4 d^{\prime}[5 / 2] 2 & 5 / 2-7 / 2 \\ & 3 / 2-5 / 2 \\ & 1 / 2-3 / 2 \\ & 7 / 2-9 / 2 \\ 4 d^{\prime}[5 / 2] 3 & 5 / 2-9 / 2 \\ & 3 / 2-9 / 2 \\ & 7 / 2-5 / 2 \\ 4 d^{\prime}[3 / 2] 2 & 5 / 2-3 / 2 \\ & 3 / 2-1 / 2 \\ & 5 / 2-3 / 2 \\ 4 d^{\prime}[3 / 2] 1 & 3 / 2-1 / 2\end{array}$

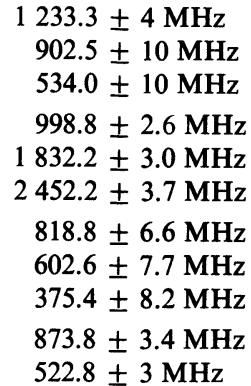

compared to the fine structure intervals in the $4 d^{\prime}$ sub-configuration, the hyperfine coupling between levels $4 d^{\prime}$ [5/2] 3, 4d $d^{\prime}$ [5/2] 2, 4d [3/2] 2 and $4 d^{\prime}$ [3/2] 1 cannot be neglected. So the various hyperfine sublevels are shifted from the position they would have if one only took into account the diagonal part of the hyperfine interaction. To correctly interpret the experimental hyperfine intervals, we have to introduce a corrective term in Casimir formula, and write the energy of sublevel $F$ of level $\alpha J$ in the following form :

$$
\begin{aligned}
E(\alpha J F) & =A(\alpha J) \frac{C_{\mathrm{F}}}{2}+ \\
& +B(\alpha J) \frac{3 C_{\mathrm{F}}\left(C_{\mathrm{F}}+1\right)-4 I(I+1) J(J+1)}{8 I(2 I-1) J(2 J-1)} \\
& +\delta E(\alpha J F)
\end{aligned}
$$

where

$$
C_{\mathrm{F}}=F(F+1)=I(I+1)-J(J+1)
$$

with $I=3 / 2$ for ${ }^{21} \mathrm{Ne}$.

The expression of $\delta E(\alpha J F)$ can be determined from second order perturbation theory with respect to the non-diagonal part of the hyperfine hamiltonian $\mathcal{H}_{\text {hyp }}$

$$
\delta E(\alpha J F)=\sum_{\substack{\alpha^{\prime} J^{\prime} \\ F^{\prime}}} \frac{\left|\left\langle\alpha J I F m_{\mathrm{F}}\left|\mathscr{H}_{\mathrm{hyp}}\right| \alpha^{\prime} J^{\prime} I F^{\prime} m_{\mathrm{F}}\right\rangle\right|^{2}}{E_{\alpha J F}-E_{\alpha^{\prime} J^{\prime} F^{\prime}}} .
$$

The summation being done over the three other states of the $4 d^{\prime}$ subconfiguration.

Using the tables of eigenstates of Neon given in [4], we see that the studied $4 \mathrm{~d}^{\prime}$ states are not very different from pure states of Racah's basis $\left({ }^{2}\right)$

$$
\left|4 \mathrm{~d}^{\prime}[K] J\right\rangle \simeq\left|j_{1}=1 / 2, \quad l=2, K, J\right\rangle .
$$

$\left({ }^{2}\right)$ Let us recall the scheme of Racah's coupling for rare gases : the core momentum $j_{1}$ (which can take the values $1 / 2$ and $3 / 2$ ) is coupled with the orbital momentum, $l$ of the external electron to give $K$, then $K$ is coupled with the spin $s$ of the external electron to give the total momentum $J$. In the $4 \mathrm{~d}^{\prime}$ levels $j_{1}$ and $K$ are almost good quantum numbers, with $j_{1}=1 / 2$ and $K=3 / 2$ or $5 / 2$.
The components of the - eigenstate labelled $\left|4 d^{\prime}[K] J\right\rangle$ on states of Racah's basis with $j_{1}^{\prime} \neq 1 / 2$ or $K^{\prime} \neq K$, as given in [4], are small and it can be shown that their contribution to $\delta E(\alpha J F)$ are negligible in comparison with our experimental precision; so for this calculation of $\delta E(\alpha J F)$, we shall only take the main component into account.

As the external electron is in a rather excited level with non-zero momentum, the hyperfine hamiltonian is only due to the interaction between the nucleus and the core electrons. Moreover, the quadrupolar hyperfine interaction has no matrix elements between states with $j_{1}=1 / 2$ so we only have to compute the matrix elements of the dipolar hamiltonian

$$
\mathscr{H}_{\mathbf{D}}=\mathbf{X} . \mathbf{I}
$$

$\mathbf{X}$ is an electronic vectorial operator, so $\mathscr{H}_{\mathbf{D}}$ is diagonal with respect to $F$ and $m_{\mathrm{F}}$.

The matrix elements involved in the calculation of $\delta E\left(4 \mathrm{~d}^{\prime}[K] J F\right)$ can finally be written as :

$$
\begin{aligned}
& \left\langle 4 \mathrm{~d}^{\prime} K_{\mathrm{F}} \operatorname{IIFm}_{\mathrm{F}}|\mathbf{X} . \mathbf{I}| 4 \mathrm{~d}^{\prime} K^{\prime} J^{\prime} I F m_{\mathrm{F}}\right\rangle= \\
& \quad=c_{1}\left(K, J, K^{\prime}, J^{\prime}, F\right)\left\langle j_{1}\|\mathbf{X}\| j_{1}\right\rangle
\end{aligned}
$$

with $j_{1}=1 / 2$.

The expression for $c_{1}$ is derived in the appendix.

On the other hand, the magnetic dipolar hyperfine constant $A\left(4 \mathrm{~d}^{\prime}[K] J\right)$ can also be expressed as a function of $\left\langle j_{1}\|\mathbf{X}\| j_{1}\right\rangle$

$$
\begin{aligned}
& A\left(4 \mathrm{~d}^{\prime}[K] J\right)= \\
= & {[J(J+1)(2 J+1)]^{-1 / 2}\left\langle 4 \mathrm{~d}^{\prime}[K] J\|\mathbf{X}\| 4 \mathrm{~d}^{\prime}[K] J\right\rangle } \\
= & c_{2}(K, J)\left\langle j_{1}\|\mathbf{X}\| j_{1}\right\rangle
\end{aligned}
$$

(see appendix for the exact expression of coefficient $c_{2}$ ).

Using the value of $A\left(4 \mathrm{~d}^{\prime}[5 / 2] 3\right)$ given by the theory of ref. [3], we obtain the value of $\left\langle j_{1}\|\mathbf{X}\| j_{1}\right\rangle$ for $j_{1}=1 / 2$ and we can then calculate the matrix elements involved in $\delta E\left(4 \mathrm{~d}^{\prime}[K] J F\right)$. The $\delta E$ values are quite important ; for example in level $4 \mathrm{~d}^{\prime}[5 / 2] 3$, the $F=3 / 2$, $F=5 / 2$ and $F=7 / 2$ are respectively shifted of $-27 \mathrm{MHz},-54 \mathrm{MHz}$ and $-54 \mathrm{MHz}$. From the so corrected hyperfine intervals, the Casimir formula enables the $A$ and $B$ values to be determined as given in table III. The last column shows the values derived from theory. As far as the magnetic structures are concerned, the agreement in fairly good for level $4 \mathrm{~d}^{\prime}$ [5/2] 3 (which justifies the value of $A\left(4 \mathrm{~d}^{\prime}[5 / 2] 3\right)$ chosen for the calculation of $\left.\left\langle j_{1}\|\mathbf{X}\| j_{1}\right\rangle\right)$ and for level $4 \mathrm{~d}^{\prime}[3 / 2] 1$; for the $J=2$ levels the agreement is not so good and we propose an interpretation of this fact in the next paragraph. For the quadrupolar part, in view of the error bars, no significant feature can be deduced from the agreement between theory and experiment.

4.2 INTERPRETATION OF THE DISAGREEMENT BETWEEN THEORY AND EXPERIMENT FOR THE $J=2$ LEVELS. - As pointed out before, the wave functions derived 
TABLE III

\section{Hyperfine structure constants}

\begin{tabular}{|c|c|c|c|c|}
\hline & $A_{\exp }(\mathrm{MHz})$ & $B_{\exp }(\mathrm{MHz})$ & $A_{\mathrm{th}}[3]$ & $B_{\mathrm{th}}[3]$ \\
\hline $4 d^{\prime}[5 / 212$ & $-355.4 \pm 1.2$ & $+8 \pm 5$ & -327.3 & \\
\hline[ & $\begin{array}{l}-235.9 \pm 0.5 \\
-236.3 \pm 0.5\left(^{1}\right)\end{array}$ & $\begin{array}{l}+11 \pm 9 \\
+6 \pm 11\left({ }^{1}\right)\end{array}$ & 9 & \\
\hline ' $[3 / 2$ & $+231.4 \pm 1.6$ & $+2 \pm 6$ & +206.1 & \\
\hline '[3/2] & $+348.3 \pm 1.6$ & $+0.8 \pm 1.7$ & +345.0 & \\
\hline
\end{tabular}

$\left({ }^{1}\right)$ These two values correspond to different ways of calculating the second order corrections; for the $A$ and $B$ values of the first line, the second order corrections have been calculated following $\S 4.1$ with the eigenstates of ref. [4]; for the second line the second order corrections have been calculated by taking into account the remark of $\S 4.2$.

in ref. [4] and used to calculate the hyperfine structures are not very different from pure states in Racah's coupling. In particular, for the $J=2$ levels :

$$
\begin{aligned}
& \left|4 d^{\prime}[5 / 2] 2\right\rangle=|1 / 2,5 / 2,2\rangle+0.032|3 / 2,5 / 2,2\rangle \\
& \left|4 d^{\prime}[3 / 2] 2\right\rangle=|1 / 2,3 / 2,2\rangle-0.062|3 / 2,3 / 2,2\rangle .
\end{aligned}
$$

(The Racah states are labelled with $\left|j_{1}, K, J\right\rangle$.)

It can be noticed that there is no mixing between states with different $K$ values. This results from the fact that the fine structure constant $\xi_{4 \mathrm{~d}}$ corresponding to the spin-orbit coupling of the external electron $\left(\xi_{4 d}\right.$ l.s) had been found to be negligible in the computer calculation. Indeed, as the two levels $4 \mathrm{~d}^{\prime}[5 / 2] 2$ and $4 d^{\prime}[3 / 2] 2$ are very near, even a weak nondiagonal interaction (such as the $4 \mathrm{~d}$ spin-orbit interaction) can mix their wave-functions. Under this hypothesis, the wave-functions of the $J=2$ levels must be written :

$$
\begin{aligned}
& \left|4 d^{\prime}[5 / 2] 2\right\rangle=(|1 / 2,5 / 2,2\rangle+ \\
& \quad+0.032|3 / 2,5 / 2,2\rangle) \cos \theta-|1 / 2,3 / 2,2\rangle \sin \theta \\
& \left|4 d^{\prime}[3 / 2] 2\right\rangle=(|1 / 2,3 / 2,2\rangle- \\
& \quad-0.062|3 / 2,3 / 2,2\rangle) \cos \theta+|1 / 2,5 / 2,2\rangle \sin \theta .
\end{aligned}
$$

We are going to show that we can determine $\theta$ from the experimental values of the hyperfine structures.

The hyperfine magnetic dipolar constant $A\left(4 \mathrm{~d}^{\prime}[5 / 2] 2\right)$ can then be expressed as follows :

$$
\begin{gathered}
A\left(4 \mathrm{~d}^{\prime}[5 / 2] 2\right)=\frac{1}{\sqrt{30}}[(\langle 1 / 2,5 / 2,2\|\mathbf{X}\| 1 / 2,5 / 2,2\rangle+ \\
+0.064\langle 1 / 2,5 / 2,2\|\mathbf{X}\| 3 / 2,5 / 2,2\rangle) \cos ^{2} \theta \\
\quad-\langle 1 / 2,5 / 2,2\|\mathbf{X}\| 1 / 2,3 / 2,2\rangle 2 \sin \theta \cos \theta \\
\left.+\langle 1 / 2,3 / 2,2\|\mathbf{X}\| 1 / 2,3 / 2,2\rangle \sin ^{2} \theta\right] .
\end{gathered}
$$

We have neglected the cross terms coming from the small components of states with $j_{1}=3 / 2$. A similar expression can be written for $A\left(4 \mathrm{~d}^{\prime}[3 / 2] 2\right)$.

Using the hyperfine interaction parameters of ref. [3] and replacing $A\left(4 \mathrm{~d}^{\prime}\right.$ [5/2] 2) and $A\left(4 \mathrm{~d}^{\prime}\right.$ [3/2] 2) by their experimental values, we obtain two determinations of $\theta$ which agree with each other : $\theta_{1}=11.6^{\circ}$ and $\theta_{2}=11.7^{\circ}$; hence the mixing coefficients are obtained

$$
\sin \theta=0.20, \quad \cos \theta=0.98 .
$$

It can easily be shown that the non-diagonal element of the spin-orbit interaction between the states $|1 / 2,5 / 2,2\rangle$ and $|1 / 2,3 / 2,2\rangle$ is $\sqrt{6} / 5 \xi_{4 d}$. Using our experimental value of the energy difference between levels $4 \mathrm{~d}^{\prime}[5 / 2] 2$ and $4 \mathrm{~d}^{\prime}[3 / 2] 2$ we deduce

$$
\xi_{4 \mathrm{~d}}=0.8 \mathrm{~cm}^{-1} \text {. }
$$

This value is small in comparison with the parameters involved in the electrostatic interaction and the spin-orbit coupling of the core. It only leads to important effects in the $4 \mathrm{~d}$ configuration because of the small energy differences between the levels. Let us note that this interpretation of the mixing of the wave-functions by the spin-orbit coupling of the external electron is only an approximation. It has been shown that other interactions (spin-spin and spin-other orbit for example) can play a role in the $4 d$ configuration [8]. Nevertheless, these interactions are always weaker than that due to spin-orbit and the order of magnitude we obtain for $\xi_{4 \mathrm{~d}}$ remains valid.

Remark. - We have just shown that the wavefunctions given in ref. [4] have to be modified by nonnegligible quantities. One can then think that the corrections $\delta E\left(4 \mathrm{~d}^{\prime}[K] J\right)$ to the hyperfine structure intervals calculated in the preceding paragraph are no longer valid and thus that the experimental hyperfine structure constants must be recalculated. In fact, one finds modifications which are small compared to the error bars except for the $4 \mathrm{~d}^{\prime}$ [5/2] 3 level for which the result is now :

$$
A=-236.3 \pm 0.5, \quad B=6 \pm 11 .
$$

5. Conclusion. - We have measured the hyperfine constants of the four levels of the $4 \mathrm{~d}^{\prime}$ subconfiguration. These measurements have provided new information concerning the excited states wave-functions and on the spin-orbit coupling in these excited states. Furthermore, the intensities of the hyperfine components provide a very accurate test of the theory of two-photon excitation. The corresponding results are presented in a following paper. 
Appendix. - As we have neglected the hyperfine interaction between the external electron and the nucleus, $\mathbf{X}$ is a vectorial operator which only acts on the core electrons; using the properties of the $6 J$ coefficients we have :

$\left\langle 4 \mathrm{~d}^{\prime} K J I F m_{\mathrm{F}}|\mathbf{X} . \mathrm{I}| 4 \mathrm{~d}^{\prime} K^{\prime} J^{\prime} I F m_{\mathrm{F}}\right\rangle=\sqrt{15}(-)^{F+1 / 2+K+K^{\prime}} \sqrt{2 K+1} \sqrt{2 K^{\prime}+1} \sqrt{2 J+1} \sqrt{2 J^{\prime}+1} \times$ $\times\left\{\begin{array}{ccc}1 / 2 & 1 & 1 / 2 \\ K^{\prime} & 2 & K\end{array}\right\}\left\{\begin{array}{lll}K & 1 & K^{\prime} \\ J^{\prime} & 1 / 2 & J\end{array}\right\}\left\{\begin{array}{lll}J & 3 / 2 & J^{\prime} \\ 3 / 2 & F & 3 / 2\end{array}\right\}\left\langle j_{1}=1 / 2\|\mathbf{X}\| j_{1}=1 / 2\right\rangle$

where we have taken $I=3 / 2$.

Similarly, the usual coupling formulas enable us to write the magnetic dipolar hyperfine constant as a function of the reduced matrix element $\left\langle j_{1}\|\mathbf{X}\| j_{1}\right\rangle$

$$
\begin{aligned}
& A\left(4 \mathrm{~d}^{\prime}[K] J\right)=[J(J+1)(2 J+1)]^{-1 / 2}\left\langle 4 \mathrm{~d}^{\prime} K J\|\mathbf{X}\| 4 \mathrm{~d}^{\prime} K J\right\rangle \simeq \\
& \simeq[J(J+1)(2 J+1)]^{-1 / 2}(-)^{J}(2 K+1)(2 J+1)\left\{\begin{array}{lll}
1 / 2 & 1 & 1 / 2 \\
K & 2 & K
\end{array}\right\}\left\{\begin{array}{lll}
K & 1 & K \\
J & 1 / 2 & J
\end{array}\right\} \times \\
& \times\left\langle j_{1}=1 / 2\|\mathbf{X}\| j_{1}=1 / 2\right\rangle
\end{aligned}
$$

which gives the coefficients $c_{1}$ and $c_{2}$.

\section{References}

[1] Biraben, F., Giacobino, E., Grynberg, G., Phys. Rev. 12A [6] Grosof, G. M., Buck, P., Lichten, W., Rabi, I. I., Phys. (1975) 2444. Rev. Lett. 1 (1958) 214

[2] Biraben, F., Grynberg, G., Giacobino, E., Bauche, J., [7] Grynberg, G., Biraben, F., Giacobino, E., Cagnac, B., Phys. Lett. 18 (1976) 374.

[3] Liberman, S., Physica 69 (1973) 598.

Opt. Commun. 18 (1976) 377.

[4] Liberman, S., Thèse Orsay (1971), unpublished.

[8] LhUILLIER, C., Thèse Paris (1976), unpublished.

[5] Biraben, F., Cagnac, B., Giacobino, E., Grynberg, G.,

[9] Moore, C. E., Atomic Energy Levels, NBS (1971). to be published. 